\title{
Computed tomography evaluation of patent paraumbilical vein and its aneurysm in relation to other portosystemic collateral channels in patients with liver cirrhosis and portal hypertension
}

\author{
Magdalena Maria Januszewicz ${ }^{A, B, C, D, E, F}$, Marta Hałaburda-Rola ${ }^{A, B, D, E, F}$, Inga Pruszyńska-Włodarczyk ${ }^{A, B, C, D, E}$, \\ Agnieszka Czachór-Zielińska ${ }^{\mathrm{A}, \mathrm{B}}$, Olgierd Rowiński ${ }^{\mathrm{A}, \mathrm{D}, \mathrm{E}, \mathrm{F}}$
}

II Department of Clinical Radiology, Medical University of Warsaw, Warsaw, Poland

\section{Abstract}

Purpose: The aim of the study was to evaluate the relationship between the diameter and aneurysmal dilatation of the paraumbilical vein (PUV) and the presence of portosystemic collateral shunts and their relationship with age and portal vein diameter.

Material and methods: The retrospective analysis, performed in the II Department of Radiology, Medical University Hospital in Warsaw, included 126 patients (77 males and 49 females) with patent umbilical vein and signs of portal hypertension due to liver cirrhosis. All patients underwent contrast enhanced abdominal CT. The average age was $54.7 \pm 12.98$. We analysed the number and type of portosystemic collateral channels in respect of age, sex, presence of oesophageal varices, and the diameter of the paraumbilical vein and the portal vein.

Results: Our results disclosed statistically significant negative correlation between patient age and diameter of paraumbilical vein, number of portosystemic collateral channels and diameter of portal vein and positive correlation between diameter of paraumbilical vein and diameter of portal vein. A statistically significant difference in diameter of portal vein and number of collateral channels was found in groups with and without oesophageal varices. No significant difference in age and portal vein diameter was found in these groups.

Conclusions: Our study showed that younger patients with liver cirrhosis are characterised by wider paraumbilical veins and higher number of portosystemic collateral channels. The presence of oesophageal varices does not correlate with age, sex, diameter of paraumbilical vein, and number of collateral portosystemic channels.

Key words: portal vein, paraumbilical vein, collateral channels.

\section{Introduction}

Increased portal pressure is the main factor leading to the formation of portosystemic collaterals, which develop by the opening, dilation, and hypertrophy of pre-existing vascular channels [1]. The most common cause of portal hypertension is the intrahepatic block caused by liver cirrhosis. Development of collateral circulation allows decompression of the portal system into systemic vasculature [2]. In advanced portal hypertension, the col- lateral circulation may carry more than $90 \%$ of the blood entering the portal system [1]. Information about collateral pathways is relevant especially when interventional procedures or surgery is considered, because accidental damage of those vessels can cause significant life-threatening bleeding [3]. The blood flow in the portal system is always dependent on pressure gradient. Typically, this is a phase-dependent, breath-dependent, hepatopetal flow, which takes place through the trunk of the portal vein - the main route of blood flow from the intestine to

Correspondence address:

Dr. Marta Hałaburda-Rola, II Department of Clinical Radiology, Medical University of Warsaw, 1 Banacha St., 02-097 Warsaw, Poland,

e-mail: mhalaburda1989@gmail.com

Authors' contribution:

A Study design - B Data collection - C Statistical analysis · D Data interpretation - E Manuscript preparation · F Literature search · G Funds collection 
the liver. Under normal conditions the small veins, called accessory portal veins, such as paraumbilical veins, gastro- hepatic vein, diaphragmatic veins, and veins of gallbladder fundus (Sappey veins), support the flow into the portal system.

It is important to remember that the portal system is not completely closed. Under normal conditions it can be powered by the inflow veins of superior vena cava and inferior vena cava, based on the current pressure gradient.

Portal hypertension $(\mathrm{PH})$, an increased blood pressure gradient between inferior vena cava and portal vein of over 5 to $10 \mathrm{mmHg}$, is a major complication in several liver diseases. The increased pressure in the portal vein (PV) causes its dilation and the formation of collateral flow.

The main interest of our study was an evaluation of collateral flow thought the paraumbilical vein (PUV). The normal falciform ligament contains one to three tiny, collapsed paraumbilical veins. In patients with cirrhosis the number and calibre of these vessels may increase [4]. The finding of a patent paraumbilical vein in the liver cirrhosis, combined with the clinical manifestation of a caput medusae in the epigastric region, is known as CruveilhierBaumgarten syndrome (CBS). The paraumbilical vein runs in the falciform ligament from the left branch of the portal vein to the anterior abdominal wall and is connected to the superior and inferior epigastric veins [5].

In portal hypertension its diameter increases, visible as a $3 \mathrm{~mm}$ or wider patent vessel, but in some cases it may be enlarged dramatically, forming a paraumbilical aneurysm. There are some indications that patent or enlarged PUV may prevent the formation of oesophageal varices, decreasing the possibility of life-threatening bleeding [5-9]. Because the PUV tends to increase blood flow to the portal trunk, the portal haemodynamics caused by developing patent PUV differ from those affected by other collateral vessels. Such a unique anatomic feature suggests that PUV may create the collateral flow, which decreases the inflow into oesophageal varices (EV). Therefore, some studies based on endoscopic evaluation of EV do not support that hypothesis and the role of PUV is not clearly established, so the opinions in the literature are divided [10-12].

There is no clear or universally accepted definition of aneurysm of the portal venous system. Some authors accept a dilated segment of a vein as an aneurysm when its diameter is significantly larger than the remaining segments of the same vessel, and when the largest diameter of that segment is beyond the accepted upper normal limit for each vessel [13].

This definition is applicable for the vessels patent in all subjects. PUV is a vessel patent only in portal hypertension, and there is no reference diameter to be applied for aneurysm assessment. Bearing that in mind, we decided to apply a value of $2 \mathrm{~cm}$ for PUV aneurysm, which is accepted in several studies for defining the aneurysm of the portal vein in the group of patients with portal hypertension [14-17].
In our interest, in the assessment based on CT scans of patients with patent PUV, we evaluated paraumbilical vein in its relation to the presence of oesophageal varices, the number of collateral channels, the portal vein diameter, and the patient's age. We made an attempt to determine risk factors of PUV aneurysm formation.

\section{Material and methods}

In our retrospective study analysed a group of 126 patients (77 male and 49 female, average age was $54.7 \pm 12.98$, median 56 years) examined in the Department of Radiology, Warsaw Medical University between the years 2007 and 2017. The database search targeted patients with patent umbilical vein and portal hypertension caused by different entities. We divided patients into two groups: with PUV $<2 \mathrm{~cm}$ (Group 1) and PUV aneurysm (Group 2).

All CT studies were performed using a 64-MDCT (Light Speed, GE), which was used according to the following protocol: three-phase CT - native, arterial and venous phase; with administration of a bolus of contrast agent (low-osmolality contrast $80 \mathrm{ml}$; injection rate $5 \mathrm{ml} / \mathrm{s}$ ).

The portal phase images used to assess the abdominal venous structures were acquired 60 seconds after a bolus contrast injection. The scanning parameters were as follows: a contiguous $2.5-\mathrm{mm}$ collimation and $12.5 \mathrm{~mm}$ per 0.5 -second table speed per $360^{\circ}$ gantry rotation, with a resultant pitch value of 1.25 . For the purpose of this analysis all studies were re-evaluated by three independent radiologists using dedicated software (Figures 1 and 2). The CT analysis consisted of the number of patent and enlarged paraumbilical veins and the type and number of collateral channels, and the diameter of portal vein and paraumbilical vein was measured. PUV appeared as a patent on CT scans as a tubular or circular enhancing structure more than $3 \mathrm{~mm}$ in diameter. We established PUV of diameter

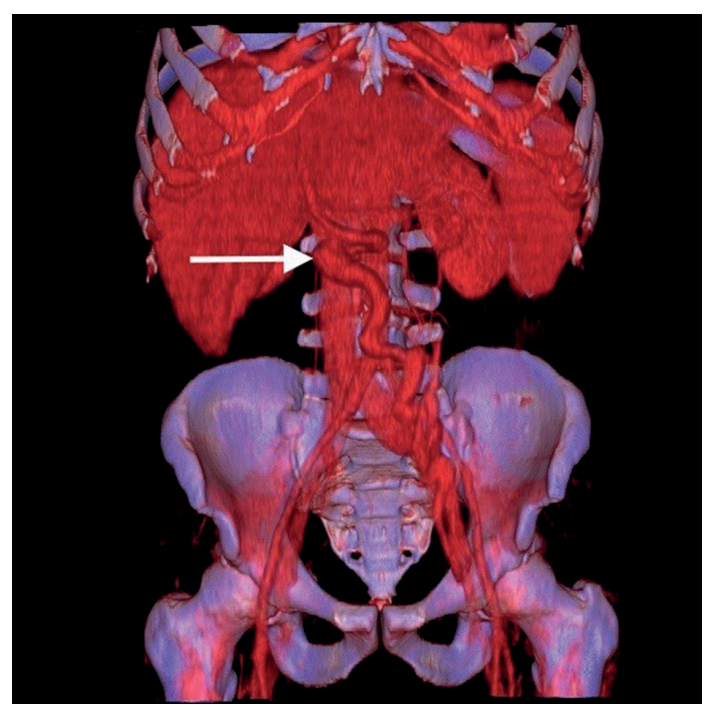

Figure 1. Volume reconstruction presenting patent, paraumbilical vein aneurysm (white arrow) with size of $22 \mathrm{~mm}$ in a 48-year-old man with liver cirrhosis 


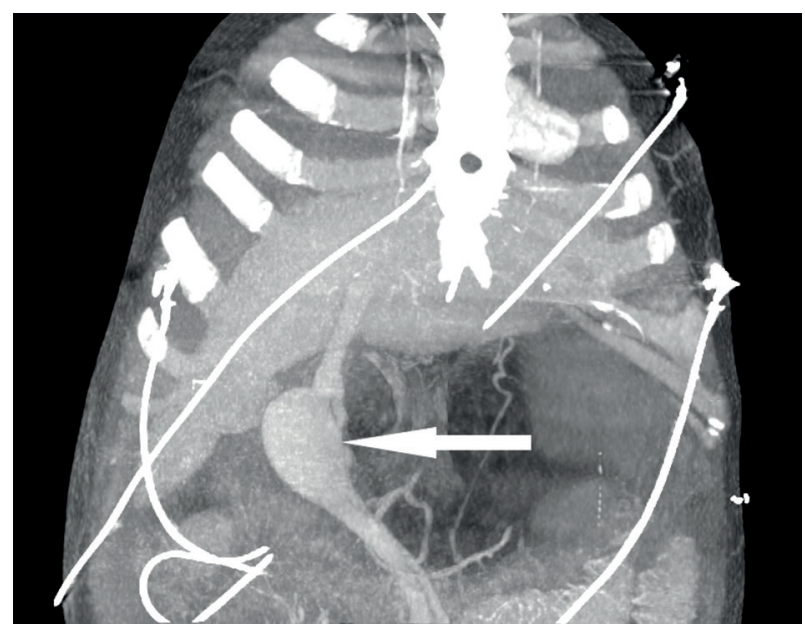

Figure 2.3D reconstruction presenting large paraumbilical vein aneurysm (white arrow) with size $42 \mathrm{~mm}$ in a 32-year-old patient with liver cirrhosis

over $20 \mathrm{~mm}$ as aneurysm and compared analysed data in two group of patients: with and without PUV aneurysm.

According to Cho et al. [18], we divided the collateral channels into 11 groups: caput medusae, coronary, oesophageal, retrogastric, perisplenic, mesenteric, omental, splenorenal, gastrorenal, abdominal wall, and retroperitoneal.

We analysed the number of collateral circulation patterns coexisting with patent paraumbilical vein.

Statistical analysis was performed with Statistica software (version 13). The Kolmogorov-Smirnov test with Lilliefors correction was used in order to estimate the normality of data (age, diameter of paraumbilical vein and portal vein); the data was not normally distributed. The Spearman correlation test was used in order to measure the strength and association between age and number of collateral channels, diameter of portal vein, and diameter of periumbilical vein.

\section{Results}

Our study showed the presence of all collateral channels in patients with patent PUV. The most frequently noticed were coronary veins (69\%), oesophageal vein (58\%), perisplenic veins (56\%), and mesenteric veins (54\%) (Figure 3). In all patient groups the portal vein mean diameter was $14.73 \mathrm{~mm}( \pm 2.706 \mathrm{~mm})$ - median $14.5 \mathrm{~mm}$. The mean diameter of the paraumbilical vein was $7.97 \mathrm{~mm}$ ( $\pm 6.02 \mathrm{~mm}$ ) ranging from $3 \mathrm{~mm}$ (patent) to $43 \mathrm{~mm}$ (aneurysm).

Our results disclosed statistically significant negative correlation between patient age and diameter of paraumbilical vein (Spearman's correlation coefficient $=-0.23$ ) (Figure 4), number of portosystemic collateral channels and diameter of portal vein (Spearman's correlation coefficient $=-0.24$ ), and positive correlation between diameter of paraumbilical vein and diameter of portal vein (Spearman's correlation coefficient $=0.28$ ) (Figures 5 and 6).

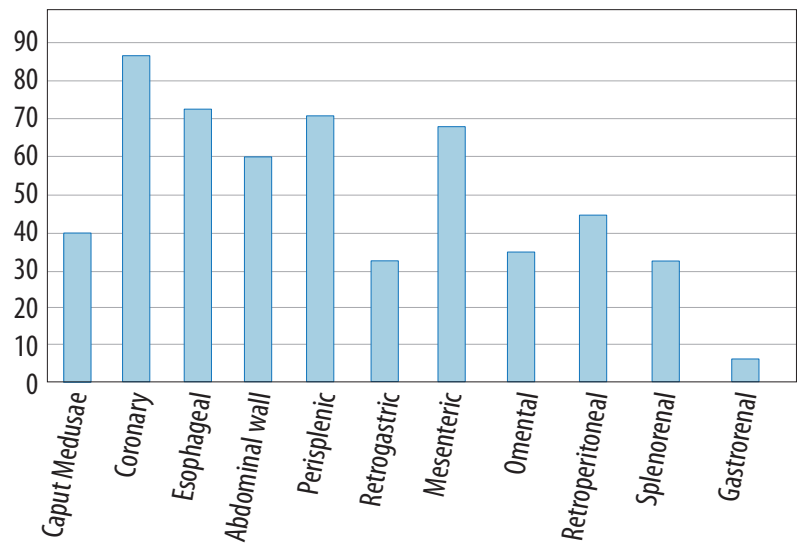

Figure 3. Distribution of vascular collateral channels

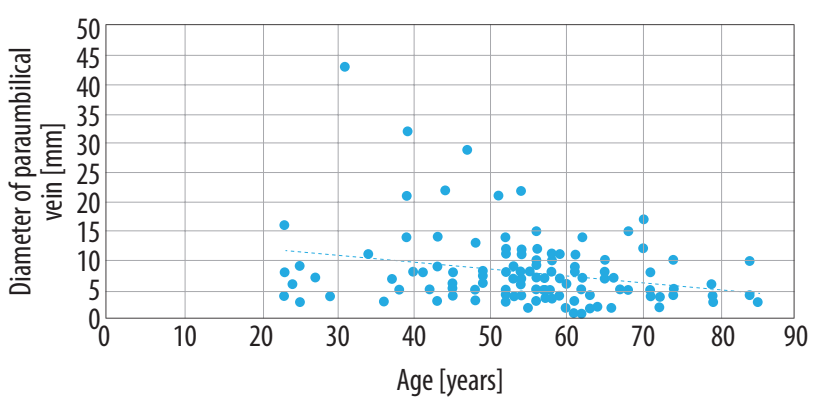

Figure 4. Diameter of paraumbilical vein vs. age

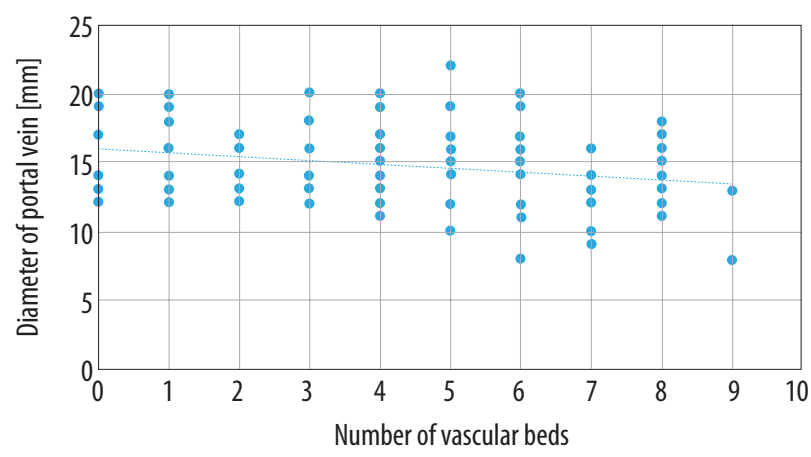

Figure 5. Number of collateral channels vs. diameter of portal vein

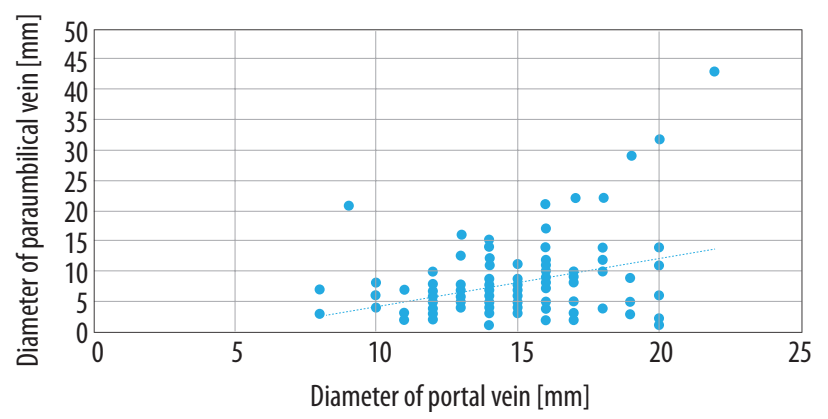

Figure 6. Diameter of paraumbilical vein and diameter of portal vein

There was a significant difference in the size of portal vein in the group with PUV aneurysm and patients without PUV aneurysm. The mean diameter of the portal vein 


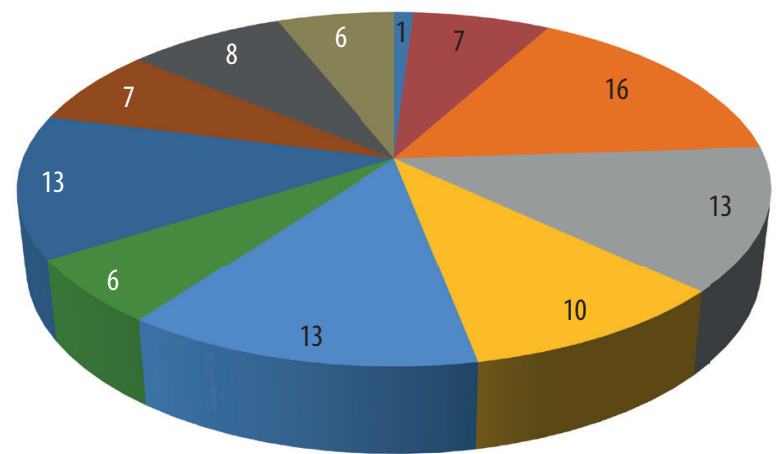

[\%]

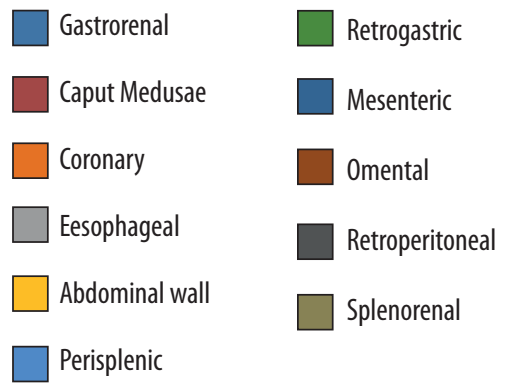

Figure 7. Number of vascular beds in group 1

in the group with PUV was $14.58 \mathrm{~mm}( \pm 2.56 \mathrm{~mm})$, and in group without PUV $17.29 \mathrm{~mm}( \pm 4.15 \mathrm{~mm}) p<0.05$. Patients with PUV aneurysm were significantly younger than those without PUV aneurysm. The mean age in group with PUV was 55.35 years ( \pm 12.99 years), and in the group without PUV 43.57 years ( \pm 7.91 years), $p<0.05$.

A statistically significant difference in diameter of portal vein and number of collateral channels was found between groups with and without oesophageal varices. The mean diameter of the portal vein in the group with oesophageal varices was $14.11( \pm 2.71)$, and in the group without oesophageal varices $15.60( \pm 2.52), p<0.05$. The mean number of collateral channels in group with oesophageal varices was $5.75( \pm 1.61)$, and in the group without oesophageal varices $2.58( \pm 1.67), p<0.05$. No significant difference in age and portal vein diameter was found in these groups.

The distribution of types and number of collateral channels in the whole group and in groups with and without PUV aneurysm are presented in Figures 7 and 8.

In the group with PUV $<2 \mathrm{~cm}$ (Group 1) and the group with PUV aneurysm the distribution of collateral channels differed in frequency of collateral flow in the anterior abdominal wall and caput medusa. The occurrence of oesophageal varices differed insignificantly in groups, with lower incidence in group 1.

\section{Discussion}

Portal hypertension is a clinical syndrome associated with increased blood pressure in the portal vein. Increased blood pressure may be caused by several entities, which can be divided into three groups depending on which part of the portal system was affected.

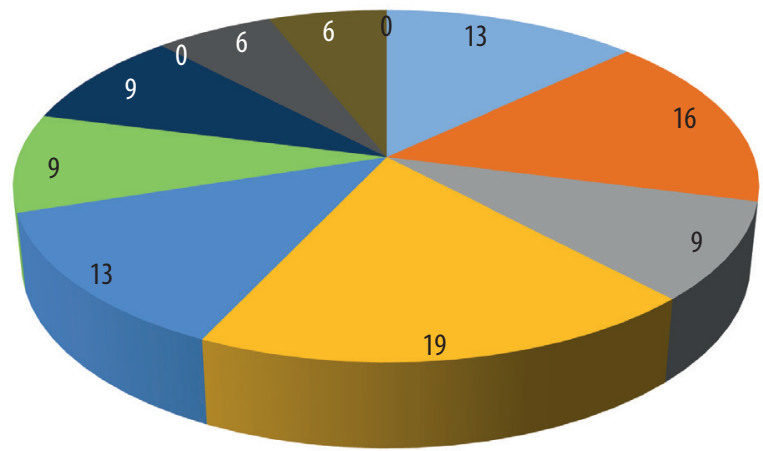

$[\%]$

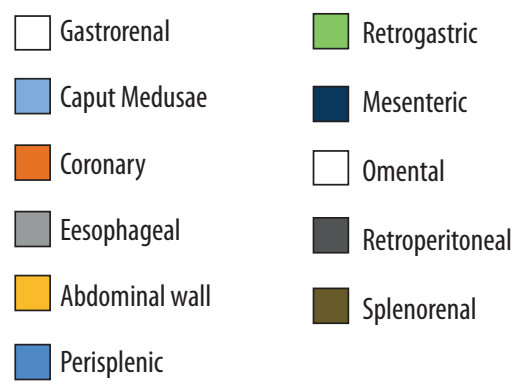

Figure 8. Number of vascular beds in group 2

The most common cause of portal hypertension in the world is liver cirrhosis - also the most common cause of portal hypertension in the analysed group of patients (98\%).

The greatest concern in the clinical course of cirrhotic patients is a bleeding from oesophageal varices. This life-threatening condition often requires immediate surgical treatment including the creation of an intrahepatic portosystemic shunt.

Patent paraumbilical vein is a common finding in patients with portal hypertension; it can be found in 6-30\% of patients with cirrhosis of the liver [19]. According to Sacerdoti et al. [20] and Chen et al. [21], patent PUV is most often present in patients with alcoholic liver cirrhosis (alcoholic vs. viral $-56 \%$ vs. $29 \%, p=0.011$ ) but the prevalence of variceal bleeding is higher, compared to patients with viral cirrhosis. In contrast, Arora et al. [7] present the view that the development of a large recanalised paraumbilical vein may prevent the bleeding from oesophageal varices but predispose to hepatic encephalopathy. Our CT study did not focus on the aetiology of liver cirrhosis, but alcoholic disease may be suspected as the dominant reason for cirrhosis.

There is some controversy in the literature regarding the relationship between PUV patency and the development varices. A duplex-Doppler study performed by Mostbeck et al. [5] in a group of 11 patients with patent PUV revealed nine patients with EV. Measuring the flow in PV and PUV, they came to the conclusion that dominant hepatofugal flow in PUV may prevent EV bleeding. Kondo et al. [12] investigated the influence of PUV patency and its effect on the portal haemodynamics by using Doppler ultrasonography. In a retrospective study on a group of 181 patients they found $26 \%$ patent PUV. 
The mean flow volume in the portal trunk, the incidence of a left gastric vein with hepatofugal flow, and the grade of the oesophageal varices were significantly higher in the patients with a patent PUV, and the deterioration of ascites during the two-year follow-up period was significantly more often in the patients with patent PUV (4/12, $33.3 \%$ ) than in those without. They concluded that patent PUV signifies pressure-loaded portal haemodynamics and a high incidence of advanced oesophageal varices in cirrhotic patients, as well as a high rate of deterioration of ascites. However, the presence of a patent PUV seems to have little effect on the disease prognosis.

In our group, oesophageal varices were present in 58\% of patients. There was no significant difference between the two groups of patients (with and without PUV aneurysm) in the presence of EV.

Portal vein aneurysm (PVA) is a very rare condition. It has been observed by US in $0.067 \%$ of patients [22]. Koc et al. [23] in a CT study reported a $0.43 \%$ prevalence of portal venous system aneurysm among 4186 patients. Lopez-Machado et al. [24], in a retrospective study using different imaging techniques in a group of 11 patients, found $13 \mathrm{PV}$ aneurysms but only one PUV, the diameter of which was $25 \mathrm{~mm}$. In an overview of international literature, Laurenzi et al. [25] presented 96 reports including 190 patients presenting portal vein aneurysm. PVA diameter in these studies was $19 \mathrm{~mm}$.

Studies concentrated on PUV aneurysm are rare. Most of them are case reports concentrating on the surgical treatment $[2,26,27]$ of haemorrhage caused by ruptured aneurysm. In a CT study by Yang [28] the retrograde analysis of 18 patients with aneurysm of umbilical portion of portal vein was performed. The diameter of aneurysm ranged from $15 \mathrm{~mm}$ to $22 \mathrm{~mm}$ (mean $18 \mathrm{~mm}$ ) and the prevalence of portal vein aneurysm of the umbilical portion was $0.109 \%$ (18 of 16,435). In our study, with the di- ameter of PUV aneurysm set at $20 \mathrm{~mm}$, the prevalence of PUV aneurysm was 5.5\% (seven from 126 pts). It should be noted that this high incidence of PUV aneurysm in comparison to other works may depend on especially selected group of patients - all with portal hypertension and patent PUV.

In our group there was a significant difference between the age of patients with and without PUV aneurysm. That may also depend on the special patient selection; other works present patients with portal hypertension, but not all of them had patent PUV [16].

Study limitations include the study protocol included only CT examination; in future protocols Doppler examination could be included for haemodynamic evaluation. Clinical data (such as etymology of liver cirrhosis) could also be used to determine possible risk factors of PUV aneurysm formation.

\section{Conclusions}

Our study showed that younger patients with liver cirrhosis are characterised by wider paraumbilical veins. Patent paraumbilical vein does not prevent the formation of oesophageal varices in the group of patients that was analysed. The patients with wider portal vein had fewer collateral channels and wider paraumbilical vein. Patients with oesophageal varices had more collateral channels and narrower portal vein than patients without oesophageal varices. We found no correlation between the number of collateral channels and the diameter of the paraumbilical vein and age.

\section{Conflict of interest}

The authors report no conflict of interest.

\section{References}

1. Bosch J, Pizcueta P, Feu F, et al. Pathophysiology of portal hypertension. Gastroenterol Clin North Am 1992; 21: 1-14.

2. Goldstein AM, Gorlick N, Gibbs D, et al. Hemoperitoneum due to spontaneous rupture of the umbilical vein. Am J Gastroenterol 1995; 1: 315-317.

3. Madrazo B, Jafri SZ, Shirkhoda A, et al. Portosystemic collaterals: evaluation with color Doppler imaging and correlation with CT and MRI. Semin Intervent Radiol 1990; 7: 169-184.

4. Lafortune M, Canostantin A, Breton G, et al. The recanalised umbilical vein in portal hypertension: a myth. AJR 1985; 144: 549-553.

5. Mostbeck GH, Wittich GR, Herold C, et al. Hemodynamic significance of the paraumbilical vein in portal hypertension: assessment with Duplex US. Radiology 1989; 170: 339-342.

6. Morin C, Lafortune M. Patent paraumbilical vein: anatomic and hemodynamic variants and their clinical importance. Radiology 1992; 185: 253-256.
7. Arora A, Rajesh S, Yamini S Meenakshi, et al. Spectrum of hepatofugal collateral pathways in portal hypertension: an illustrated radiological review. Insights Imaging 2015; 6: 559-572.

8. Gallego C, Velasco M, Marcuello P, et al. Congenital and acquired anomalies of the portal venous system. Radiographics 2002; 22: 141-159.

9. Zardi EM, Uwechie V, Caccavo D, et al. Portosystemic shunts in a large cohort of patients with liver cirrhosis: detection rate and clinical relevance. J Gastroenterol 2009; 44: 76-83.

10. Ditchfield MR, Gibson RN, Donlan JD, et al. Duplex Doppler ultrasound signs of portal hypertension: relative diagnostic value of examination of paraumbilical vein, portal vein and spleen. Australas Radiol 1992; 36: 102-105.

11. Gibson RN, Gibson PR, Donlan ID, et al. Identification of a patent paraumbilical vein by using Doppler sonography: importance in the diagnosis of portal hypertension. AJR Am J Roentgenol 1989; 153: 513-516. 
12. Kondo T, Maruyama H, Sekimoto T, et al. Influence of paraumbilical vein patency on the portal hemodynamics of patients with cirrhosis. J Clin Gastroenterol 2014; 48: 178-183.

13. Ozbek SS, Killi MR, Pourbagher MA, et al. Portal venous system aneurysms: report of five cases. J Ultrasound Med 1999; 18: 417-422.

14. Elsayes KM, Shaaban AM, Rothan SM, et al. A comprehensive approach to hepatic vascular disease. Radiographics 2017; 37: 813-836

15. Giannoukas A, Sfryroeras G. Current management of visceral venous aneurysms. Phlebolymphology 2010; 17: 130.

16. Koç Z, Oğuzkurt L, Ulusan S. Portal venous system aneurysms: imaging, clinical findings and a possible new etiological factors. AJR Am J Roentgenol 2007; 189: 1023-1030.

17. Yang DM, Yoon MH, Kim HS, et al. Portal vein aneurysm of the umbilical portion: imaging features and the relationship with portal vein anomalies. Abdom Imaging 2003; 28: 62-67.

18. Cho KC, Patel YD, Wachsberg RH, et al. Varices in portal hypertension: evaluation with CT. Radiographics 1995; 15: 609-633.

19. Dökmeci AK, Kimura K, Matsutani S, et al. Collateral veins in portal hypertension: demonstration by sonography. AJR Am J Roentgenol 1981; 137: 1173-1177.

20. Sacerdoti D, Bolognesi M, Bombonato G, et al. Paraumbilical vein patency in cirrhosis: Effects on hepatic hemodynamics evaluated by Doppler sonography. Hepatology 1995; 22: 1689-1694.
21. Chen $\mathrm{CH}$, Wang JH, Lu SN, et al. Comparison of prevalence for paraumbilical vein patency in patients with viral and alcoholic liver cirrhosis. Am J Gastroenterol 2002; 97: 2415-2418.

22. Ohnishi K, Nakayama T, Saito M, et al. Aneurysm of the intrahepatic branch of the portal vein. Report of two cases. Gastroenterology 1984; 86: 169-173.

23. Koç Z, Oğuzkurt L, Ulusan S. Portal vein variations: clinical implications and frequencies in routine abdominal multidetector CT. Diagn Interv Radiol 2007; 13: 75-80.

24. Lopez-Machado E, Mallorquin-Jimenez F, Medina-Benitez A, et al. Aneurysms of the portal venous system: ultrasonography and CT findings. Eur J Radiol 1998; 26: 210-214.

25. Laurenzi A, Ettorre GM, Lionetti R, et al. Portal vein aneurysm: What to know. Dig Liver Dis 2015; 47: 918-923.

26. Kim PTW, Chandy TT, Ghanekar A. Massive enlargement of a paraumbilical vein in a patient with advanced portal hypertension. Surgery 2012; 152: 931-933.

27. Lewis CP, Murthy S, Webber SM, et al. Hemorrhage from recanalized umbilical vein in a patient with cirrhosis. Am J Gastroenterol 1999; 94: 280.

28. Yang DM, Yoon MH, Kim HS, et al. Portal vein aneurysm of the umbilical portion: imaging features and the relationship with portal vein anomalies. Abdom Imaging 2003; 28: 62-67. 